\title{
The GB6 4.85 GHz Radio Variability Catalog
}

\author{
P. C. Gregory, P. Capak, D. Gasson
}

Physics $\&$ Astronomy, University of British Columbia, Vancouver, B.C., V6T 1Z1, Canada

\section{W. K. Scott}

Physics and Astronomy, University of Calgary, Calgary, Alberta, T2N 1N4, Canada

\begin{abstract}
The NRAO seven-beam receiver was used on the former Green Bank $91 \mathrm{~m}$ telescope, during 1986 November and 1987 October, to repeatedly survey the declination band $\left(0^{\circ}<\delta<+75^{\circ}\right)$ at $4.85 \mathrm{GHz}$. This data base has been used to extract variability information for sources in the GB6 catalog, a large unbiased sample of sources derived from the combined survey images. Long term variability information (one year time base) is available for $97 \%$ of the 75,162 GB6 sources and short term variability (few day) is available for 20,963 sources. This large variability data base is available to the astronomical community through http://pulsar.physics.ubc.ca/gregory/index.html.
\end{abstract}

\section{Introduction}

One way to discover and probe the nuclear regions of active galaxies is through a study of radio variability. The Green Bank $4.85 \mathrm{GHz}$ survey was carried out with following three types of data products in mind. a) A set of sky maps (Condon et al. $1991 \&$ 1994). b) These sky maps were used to construct catalogs of compact radio sources (Gregory \& Condon 1991; Gregory et al. 1996). The GB6 catalog of 75,162 discrete sources, with angular sizes $\leq 10.5$ arcmin and flux densities $\geq 18 \mathrm{mJy}$, was based on the sky maps constructed from both epochs. c) The final goal was to extract limited variability information for the entire catalog of GB6 sources. This variability information consists of two parts. First, there is an indicator of the long term variability, based on two flux density measurements, one derived from the 1986 image set and the other from the 1987 image set. Second, there is an indicator of the short term variability, based on an analysis of the daily scan data that was used to construct the images. Long term variability information is available for $97 \%$ of the 75,162 GB6 sources. It has been possible to derive short term variability information for 20,963 GB6 sources. The exact number of daily measurements depends on source declination, ranging from a mean of 7 (both epochs) for a source at declination $0^{\circ}$ to a mean of 20 for a declination of $70^{\circ}$.

Figure 1 shows the number of sources for which $\mathrm{N}$ daily flux density measurements were obtained as a function of N. Figure 2 shows an example of the 


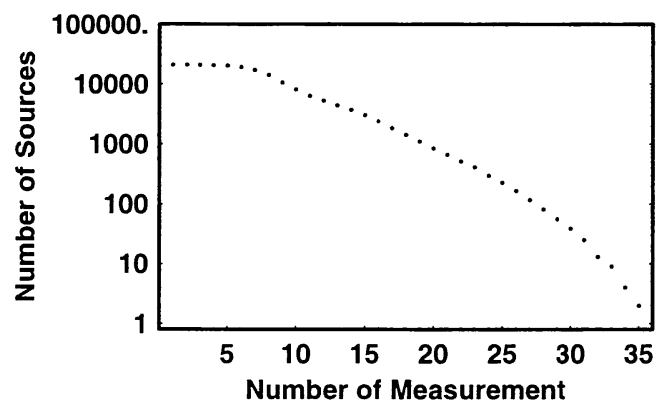

Figure 1. The number of sources for which $\mathrm{N}$ daily flux density measurements were obtained as a function of $\mathrm{N}$.
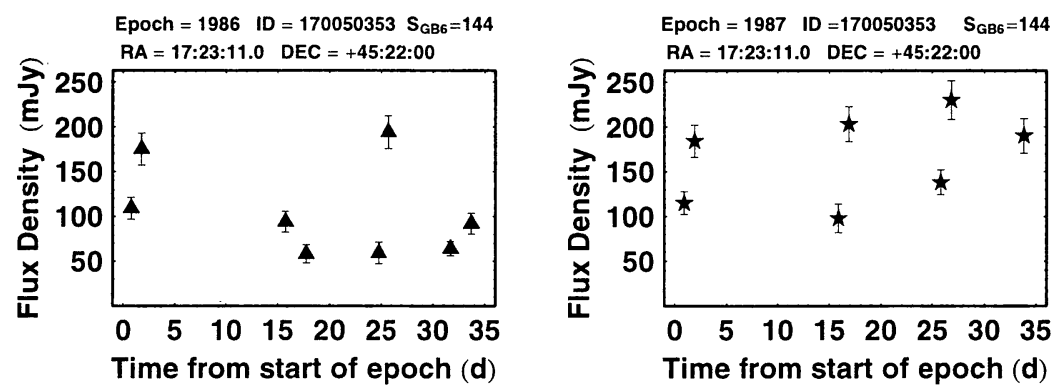

Figure 2. Sample short term variable source.

daily flux density measurements for a GB6 source which exhibits short term variability in both epochs. Details of the analysis of the long and short term variability will appear shortly in Scott and Gregory (in preparation 2000), and Gregory et al. (in preparation 2000).

Acknowledgments. This research was supported in part by a grant from the Natural Science and Engineering Research Council of Canada.

\section{References}

Condon, J. J., Broderick, J. J., \& Seielstad, G. A. 1989 in AJ97, 1064

Condon, J.J., Broderick, J.J., Seielstad, G.A., Douglas, K., \& Gregory, P.C. 1994 in AJ107, 1829

Gregory, P.C. \& Condon, J.J. 1991 in ApJS75, 1011

Gregory, P.C., Scott, W.K., Douglas, K., \& Condon, J.J. in ApJS103, 427 